\title{
Muscle strength after the anterior cruciate ligament reconstruction via contralateral bone- tendon-bone autograft
}

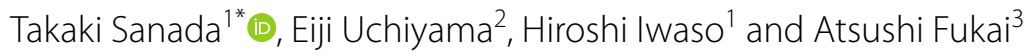

\begin{abstract}
Purpose: The anterior cruciate ligament (ACL) reconstruction via a contralateral bone-tendon-bone (C-BTB) autograft was introduced to encourage early return to sports. The purpose of this study is to evaluate whether primary contralateral BTB ACL reconstruction can be adapted for early return-to-sports modification by investigating the chronological changes of muscle strength after surgery.

Methods: Fifteen patients who had underwent C-BTB ACL reconstruction were compared with a matched group of 15 patients of ipsilateral BTB (I-BTB) ACL reconstruction. The clinical outcomes of the time of return-to-sports, Tegner activity scale and the rate of second $\mathrm{ACL}$ injuries, the tibial anterior translation measurement, and knee extension and flexion muscle strength were assessed.

Results: Within 12 months after surgery, 14 of 15 patients from both groups returned to preinjury sports. The median time to return to sports after surgery was 6.5 months in the C-BTB group and 8.0 months in the I-BTB group $(p=0.021)$. No significant difference was noted with regard to the Tegner activity scale, reinjury rate or mean instrumental anterior tibial translation. The quadriceps muscle strength in the ACL-reconstructed knee compared with the opposite knee in both groups at 5 months after surgery was $120.6 \%$ in the C-BTB group and $70.0 \%$ in the I-BTB group $(p<0.001)$. However, the quadriceps muscle strength of the non-reconstructed limb, which instructed the graft harvested knee in the C-BTB and the intact knee in the I-BTB group, compared with that of the preoperative uninjured $\operatorname{limb}$, was $74.5 \%$ in the C-BTB group and $118.7 \%$ in the I-BTB group $(p=0.0021) 5$ months after surgery. Moreover, the quadriceps muscle strength of the reconstructed knee compared with the preoperative normal limb was $88.8 \%$ and $81.5 \%$ in the C-BTB and I-BTB groups, respectively $(p=0.38)$.
\end{abstract}

Conclusions: $A C L$ reconstruction via the $C-B T B$ autograft indicated better quadriceps muscle strength from early stage after surgery compared with I-BTB ACL reconstruction. However, the ostensible rapid symmetrical muscle strength recovery was attributed to strength deficits compared to the preoperative condition at the donor site limb and ACL-reconstructed limb.

Level of evidence: Level: Level: 4.

Keywords: Anterior cruciate ligament, Anterior cruciate ligament reconstruction, Bone-tendon-bone, Contralateral, Contralateral graft, Contralateral reconstruction, Quadriceps muscle, Return to sports

\footnotetext{
*Correspondence: sanasana511@gmail.com

1 Department of Sports Orthopaedic Surgery, Kanto Rosai Hospital, Kanto Rosai Hospital, 1-1 Kizuki Sumiyoshicho, Nakahara-Ku, Kawasaki, Kanagawa Prefecture 211-8510, Japan

Full list of author information is available at the end of the article
}

\section{Introduction}

Recovery from anterior cruciate ligament (ACL) injuries in athletes, especially at pre-injury levels, is often difficult. The overall return-to-play rate ranges from $60 \%$ to $80 \%$ in various sports [7]. Furthermore, only $63 \%$ of the 
patients re-engage in their preinjury sports, and only $44 \%$ among competitive athletes did [1]. Consequently, it takes 6-13 months for these patients and athletes to return to sports after ACL reconstruction [9]. Generally, ACL rupture is a season-ending injury for athletes since it requires a long time for them to recover and return to playing sports.

Early return-to-play, that even increases a risk of the rate of sustaining a second ACL injury [2], is often a matter of consequence among young athletes who survive in competitive teams. To shorten the return-to-play time successfully, Shelbourne et al. developed contralateral BTB ACL reconstruction [14]. This procedure results in accelerated rehabilitation that allows patients to recover fully and return to playing sports in approximately 4-6 months after reconstruction $[5,14,17]$. The main concept of the procedure is to secure better quadriceps muscle strength by harvesting a bone-patellar tendon-bone graft from the unaffected limb, which divides the duplicative antagonistic effects of reconstruction and graft harvesting maneuvers on one limb into two separate knees. This earlier return-to-sports strategy is supported by the biological healing advantage of BTB autografts, which mature faster than hamstring grafts [6].

In the return-to-play criteria, $86 \%$ of the studies used isokinetic muscle strength tests and symmetrical quadriceps strength recovery more than $80 \%$ of that of the opposite limb set as the goal after ACL reconstruction $[4,12]$. According to a previous study on the muscle strength measurement on the contralateral BTB ACL reconstruction, the patients who underwent contralateral BTB ACL reconstruction secured better quadriceps muscle strength than ipsilateral BTB ACL reconstruction at 2 years postoperatively [13]. However, no report on contralateral BTB reconstruction described muscle strength in the early period when the patients returned to play.

The purpose of the study was to evaluate whether primary contralateral BTB ACL reconstruction can be adapted for early return-to-sports modification by investigating the chronological changes of muscle strength after surgery.

\section{Materials and methods}

This study was approved by the institutional review board of Kanto Rosai Hospital. The ID number was 2019-9. Informed consent for responding to treatment was obtained from each patient by the responding surgeon, and participant agreement in the clinical research was conducted with opt-out approval.

Between 2011 and 2014, 363 patients underwent ACL reconstruction via the autograft of the bone-patellar tendon-bone in our institution. After excluding 167 revision ACL surgeries, 18 bilateral ACL reconstructions, and 15 knees with multiple ligaments, the remaining 163 primary ACL reconstruction via the transtibial tunnel creation technique were included in the investigation. Of 163 primary BTB reconstruction knees, 15 athletes underwent $A C L$ reconstruction surgeries using the contralateral BTB autograft (C-BTB) and 148 knees underwent $A C L$ reconstruction with the ipsilateral $B T B$ autograft (I-BTB). The selection for the surgical procedure via the contralateral BTB graft was determined under adequate discussions between the surgeon and the patients who wished to return to their previous sports in an earlier period. To compare the two different procedures, 15 knees from 148 primary ipsilateral BTB reconstruction patients were selected as the control matched group to decrease preoperative bias. The groups were matched for sex, age, type of sports activity, Tegner activity scale, and incident of concomitant meniscus injuries (Table 1). The mean follow-up periods were 14.9 months in the C-BTB group and 20.9 months in the I-BTB group.

We compared the median return-to-sports times between the two groups. The median postoperative Tegner activity scale, incidence of second ACL injuries, and the mean tibial anterior translational distance difference between both knees using the KT-2000 arthrometer (MedMetric Inc., San Diego, CA, USA), and quadriceps and hamstring muscle strengths between the two groups were investigated. In this study, we defined return to sports as participation in the normal practice of the whole team on their previous team or another team. Muscle strength was compared in three different evaluation methods using the Biodex System 3 isokinetic dynamometer (Biodex Medical Systems, New York, USA) at anglar velocities of $60^{\circ} / \mathrm{s}$. First, the quadriceps and hamstring muscle strength of the reconstructed knee compared with the opposite knee were investigated. Then, the chronological muscle strength change of the reconstructed knee was measured by comparing it with the preoperative uninvolved normal knee. Third, the chronological change in muscle strength of the non-reconstructed knees, including the graft harvested donor knee in the C-BTB group and the uninvolved intact knee in the I-BTB group, was investigated. Preoperative muscle strength was measured at the day before surgery. Muscle strength was measured at the following three periods: preoperative, 5 months after surgery, and 12 months after surgery.

Reconstruction surgeries were performed using the central one-third of the bone-patellar tendon-bone autograft. The BTB grafts were harvested from the ipsilateral knee in the I-BTB group and from the contralateral knee in the C-BTB group. A single round tibial tunnel was created first; then, a round femoral tunnel socket was created via a transtibial approach. The bone-patellar 
Table 1 Matched patients' baseline characteristics

\begin{tabular}{|c|c|c|c|}
\hline Group & $\begin{array}{l}\text { Contralateral BTB } \\
(n=15)\end{array}$ & $\begin{array}{l}\text { Ipsilateral BTB } \\
(n=15)\end{array}$ & $p$ value \\
\hline Age (y) (mean [ min.-max.]) & $20.2[16-36]$ & $19.7[14-27]$ & 0.74 \\
\hline Height (cm) (mean [ min.-max.]) & $175.1[166-186]$ & $174.1[163-183]$ & 0.7 \\
\hline Weight (kg) (mean [ min.-max.]) & $75.9[53-115]$ & $75.7[50-105]$ & 0.98 \\
\hline \multicolumn{4}{|l|}{$\operatorname{Sex}(n(\%))$} \\
\hline Female & $4(26.7)$ & $3(20)$ & \multirow[t]{2}{*}{0.67} \\
\hline Male & $11(73.3)$ & $12(80)$ & \\
\hline \multicolumn{4}{|l|}{ Sports (n (\%)) } \\
\hline American football & $4(26.7)$ & $4(26.7)$ & \multirow[t]{5}{*}{0.731} \\
\hline Soccer & $1(6.7)$ & $2(13.3)$ & \\
\hline Basketball & $4(26.7)$ & $4(26.7)$ & \\
\hline Rugby & $4(26.7)$ & $4(26.7)$ & \\
\hline Snowboard & $1(6.7)$ & $0(0.0)$ & \\
\hline Tegner activity scale (median [ min.-max.]) & $9[6-10]$ & $9[6-10]$ & 1 \\
\hline Concomitant meniscal injuries (n (\%)) & $4(26.7)$ & $3(20.0)$ & 0.67 \\
\hline
\end{tabular}

BTB Bone-tendon-bone

tendon-bone autograft was fixed by the suspension device of the ENDOBUTTON (Smith and Nephew, Inc., Andover, MA, USA) on the femoral cortex and tied to the tibial post fixation screw and washer (MeiraGTx Limited, Nagoya, Japan). All autografts were fixed at the full knee extension position while applying adequate tension to the graft by hand.

Postoperative rehabilitation was performed using the same protocol for both groups. Physical therapy was initiated on postoperative day 1 , with the patients wearing a hinged knee orthosis. Once tolerated, the patients started walking with full weight bearing. The range of motion exercise was started to aim for an immediate full extension knee from the following day and gradual flexion to obtain full flexion at 4 months after surgery. Jog training began 2 months postoperatively. Agility exercises and open kinetic muscle training were started 4 months after surgery. At 5 months postoperatively, after estimating isokinetic knee extension and flexion muscle strength, the patients participated in sports activities aiming at returning to the competitive level when they have met the goal set for quadriceps and hamstring muscle recovery. We set the goal of muscle strength to be over $80 \%$ knee extension and flexion peak torque of the reconstructed knee compared with that of the contralateral extremity using the Biodex System 3 isokinetic dynamometer.

For statistical analysis, we used EZR (Jichi Medical University, Saitama Medical Center, Saitama, Japan), which is a modified version of $\mathrm{R}$ (The R Foundation, Vienna, Austria) for 1:1 matching data extraction from the pooled control group and comparison datasets between the two groups. Then, we used the Mann-Whitney U test to compare the groups in ordinal and continuous variables and chi-square test for the categorical variables with Excel statistical analysis (BellCurve, version 3.21). We defined $p$-values of $<0.05$ as being statistically significant in the series.

\section{Results}

The return-to-play time was significantly earlier in the C-BTB group than in the I-BTB group $(6.5$ months in the C-BTB group vs. 8.0 months in the I-BTB group; $p=0.021$ ). Clinical outcomes at 1 year after surgery were not significant different according to the Tegner activity scales and the tibial anterior translation measured using the KT-2000 arthrometer. Concerning the occurrence rates of second ACL injuries, graft reinjury was $6.7 \%$ $(1 / 15)$ in both groups, and the contralateral ACL injury was $6.7 \%(1 / 15)$ in the I-BTB group, with none of the patients in the C-BTB group deploying this injury within the follow-up period (Table 2). Concerning the comparing muscle strength between the reconstructed knee and un-reconstructed knee, the quadriceps muscle strength in the C-BTB group is significantly higher than that in the I-BTB group at 5 months and 12 months postoperatively. In contrast, hamstring muscle strength was not significantly different between the groups (Fig. 1). The postoperative chronological quadriceps and hamstring muscle strength changes in the reconstructed knees compared to the preoperative normal limb were not significantly different between the two groups (Fig. 2). However, muscle strength in non-reconstructed knees (graft donor knee in the C-BTB group and uninvolved intact knee in the I-BTB group) compared to the preoperative normal 
Table 2 Clinical data after surgery

\begin{tabular}{|c|c|c|c|c|}
\hline \multicolumn{2}{|l|}{ Group } & $\begin{array}{l}\text { Contralateral BTB } \\
(n=15)\end{array}$ & $\begin{array}{l}\text { Ipsilateral BTB } \\
(n=15)\end{array}$ & $p$ value \\
\hline Time of return-to-sports & (median [ min.-max.]) & $6.5[5-8.5]$ & $8[6-11]$ & 0.012 \\
\hline Tegner activity scale & (median [ min.-max.]) & $9[6-10]$ & $9[6-10]$ & 1 \\
\hline \multicolumn{5}{|l|}{ Lachman } \\
\hline negative & $(\mathrm{n}(\%))$ & $13(86.7)$ & $13(86.7)$ & 1 \\
\hline glide & & $2(13.3)$ & $2(13.3)$ & \\
\hline gross & & $0(0)$ & $0(0)$ & \\
\hline \multicolumn{5}{|l|}{ Pivot shift } \\
\hline negative & $(\mathrm{n}(\%))$ & $12(80.0)$ & $12(80.0)$ & 1 \\
\hline glide & & $2(13.3)$ & $2(13.3)$ & \\
\hline gross & & $1(6.7)$ & $1(6.7)$ & \\
\hline Anteror translation differenc & (mean \pm SD) & $1.3 \pm 1.2$ & $1.9 \pm 1.9$ & 0.36 \\
\hline \multicolumn{5}{|l|}{ Second $A C L$ injuries } \\
\hline graft re-rupture & $(\mathrm{n}(\%))$ & $1(6.7)$ & $1(6.7)$ & \\
\hline contralateral $\mathrm{ACL}$ injuries & $(\mathrm{n}(\%))$ & $0(0)$ & $1(6.7)$ & \\
\hline
\end{tabular}

BTB Bone-tendon-bone

limb was significantly weaker in the C-BTB group than in the I-BTB group at 5 months $(p=0.0021)$ and 12 months $(p=0.023)$ after surgery in the quadriceps. In contrast, hamstring muscle strength was not significantly different at either 5 months $(p=0.33)$ or 12 months $(p=0.74)$ after surgery (Fig. 3$)$. Twenty percentage (3/15) of the patients in the C-BTB group required local anesthetic injection therapy before or after return to playing sports due to the graft harvested site pain, yet none of the patients in the I-BTB group required anesthetic injections $(p=0.069)$.

\section{Discussion}

The most important aspect of this study was to investigate postoperative muscle strength whether the contralateral BTB reconstruction meets the early returnto-sports criteria. To date, no previous reports have referred to muscle strength at the early stages within 1 year after surgery of $\mathrm{C}$-BTB reconstruction. In this study, the patients who underwent C-BTB ACL reconstruction shown that the $120.6 \%$ of quadriceps and $95.6 \%$ of hamstring muscles strength of the reconstructed knee compared with the non-reconstructed knee at 5 months after surgery. From this rapid muscle strength recovery, the C-BTB approach may superficially seems to be an acceptable modification to ACL reconstruction to allow patients to return to play sports in the early stages. However, we note that the quadriceps muscle strength of the non-injured knee decreased by $74.5 \%$ in the graft harvested knee and $88.8 \%$ in the ACL-reconstructed knee at the same time. Early muscle strength recovery of the reconstructed knee compared to the none-reconstructed knee is due to bilateral quadriceps muscle strength deficits. We doubt that this technical modification secures a safe early return to sports with bilateral quadriceps muscle weakness. From the results, we have not attempted primary ACL reconstruction using a BTB autograft from the contralateral knee since 2015.

In general, the concept of rehabilitation in patients who underwent ACL reconstruction has antagonistic effects. One is the temporal postoperative rest of the reconstructed knee to promote graft and bone tunnel healing. The other is early knee ROM exercises and muscle training to build up to the preoperative condition and prevent graft site morbidities. Theoretically, the $\mathrm{C}$-BTB ACL reconstruction has an advantage over the conventional I-BTB ACL reconstruction by facilitating postoperative rehabilitation by dividing the duplicative antagonistic effect on a single knee into two separate knees. Shelbourne et al. developed the advantages of the C-BTB approach of ACL reconstruction to allow patients to return to playing sports earlier by restoring ROM and muscle strength $[14,16]$. Benner et al. also reported the advantages of the C-BTB approach in terms of postoperative complications [3]. They concluded that the incidence of infection or patellar tendon rupture was not different between the ipsilateral and contralateral BTB reconstruction groups. However, patients with complications after ACL reconstruction in the contralateral group may have less difficulty in obtaining full ROM than those in the ipsilateral reconstruction group.

In contrast, Mastrokalos et al. [10] reported that contralateral grafts appeared to offer no significant advantages over ipsilateral grafts. There was no tibial 


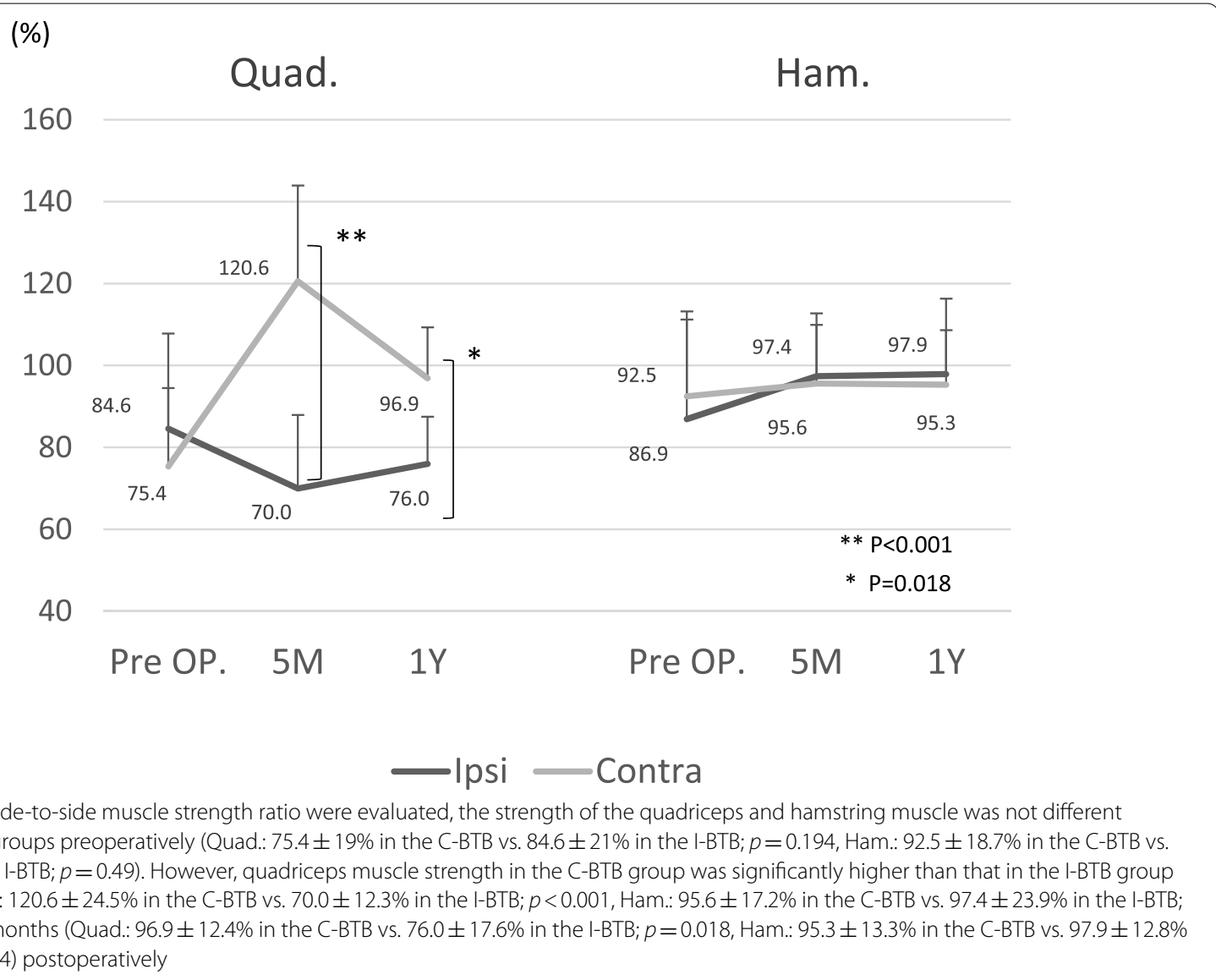

translation difference measured using the KT-2000 arthrometer and no difference in the Cincinnati Knee Rating System and Tegner activity scores. In thier contralateral BTB group, $70.9 \%$ of the patients experienced kneeling pain in the donor knee, whereas in their ipsilateral BTB group, $69.2 \%$ of the patients experienced kneeling pain in the donor knee, showing no significant difference between the two groups. In addition, contralateral ACL reconstruction did not result in a shorter return-to-play time compared to ipsilateral ACL reconstruction. Therefore, they concluded that the symptoms related to morbidity of the donor site pain was transferred to the uninvolved knee without any advantages.

With regard to the graft site pain in this study, 20\% of the patients in the C-BTB group required anesthetic injections at the donor site to control anterior knee pain when they returned to playing sports; in contrast, none of the patients in the I-BTB group required anesthetic injection therapy. Mastrokalos et al. [10] evaluated the postoperative donor site morbidity. Kneeling pain in the donor knee was experienced by $69.2 \%$ and $70.9 \%$ of the patients in the ipsilateral and contralateral BTB groups, respectively. Although the overall comparison of the donor site pain rates between the two groups was not statistically significant, in the subgroup analysis, the rate of severe kneeling pain in the donor knee in the patients in the contralateral BTB group was significantly higher (27.1\%) than that in the ipsilateral BTB group (9.6\%) $(p=0.02)$. Rubinstein et al. [14], referring to the donor site morbidity using contralateral BTB grafts in mostly revision ACL reconstruction cases, reported that the most common complication was intensive donor site patellar tendon pain. They added that the transient pain was unresolved even at 1 year after surgery, while postoperative donor site pain decreased sequentially.

In contrast, Shelbourne et al. reported that no difference in kneeling and severity of pain in the International Knee Documentation Committee Subjective Knee Form subscores between the contralateral and ipsilateral BTB groups $(p=0.686$ in kneeling and $p=0.564$ in severity of pain) $[13,15]$. They concluded that failure to prevent anterior knee pain was mostly due to extension deficit of the knee during the preoperative and postoperative periods $[13,15]$. Although we also focused on the ROM exercise and muscle training from pre- to postoperative rehabilitation to minimize extension deficit, thus 


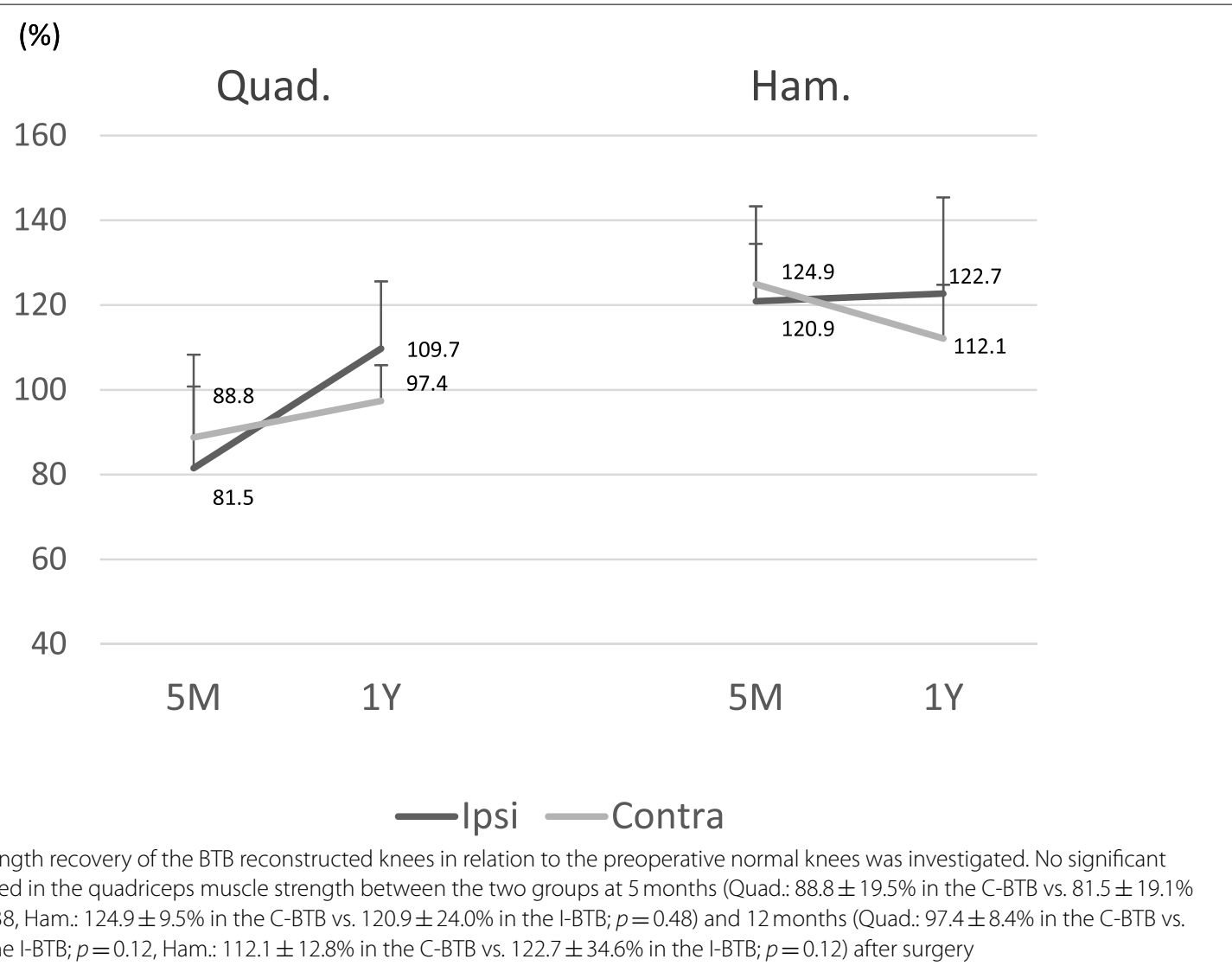

preventing anterior knee pain according to this recommendation, $20 \%$ of the contralateral BTB reconstruction still required local anesthetic injection therapy to reduce severe knee pain. We believe that the knee pain linked to the quadriceps muscle weakness is a significant reason for the donor site knee in the contralateral $\mathrm{BTB}$ reconstruction as the non-ACL injured limb should be the dominant leg in the early stage of return to sports. For these reasons, we have abandoned the adaptation of the contralateral BTB graft modification to achieve early return to sports since 2015.

As another approach to reduce donor site pain, the technique of refilling with a substitute material into the bony defect after harvesting the BTB graft was introduced $[8,11]$. Refilling the void with a substitute material reduced graft site kneeling pain and postoperative patellar crepitus compared to non-refilling techniques. A welldesigned comprehensive rehabilitation program and graft harvesting technique will be useful for reducing pain.

As with all studies, the limitations of this study exist. First, this was a retrospective study with a small number of patients. Fifteen patients apparently lacked power for the analysis. However, because no patients have been performed using this modification technique presently, the study has stopped as a pilot study that had implied the limitation of this technique. Moreover, the small number of patients did not increase the risk of second ACL injuries that should be critically evaluated. Second, with regard to the donor site morbidity as a result of bone-patellar tendon-bone autograft harvesting, graft site pain is evaluated only based on the frequency of the injection treatments at the site. We did not compare the C-BTB group with the I-BTB group using a subjective or objective knee score. However, to the best of our knowledge, no other studies have evaluated quadriceps and hamstring muscle strength in the early period when the patients anticipated going back to their previous sports. This information will guide surgeons and patients when they prefer accelerated rehabilitation after ACL reconstruction via contralateral BTB autografts.

\section{Conclusions}

ACL reconstruction via the $\mathrm{C}-\mathrm{BTB}$ autograft indicated better quadriceps muscle strength from early stage after surgery compared with I-BTB ACL reconstruction. However, we should consider that muscle strength symmetry can result from simultaneous bilateral knee muscle 


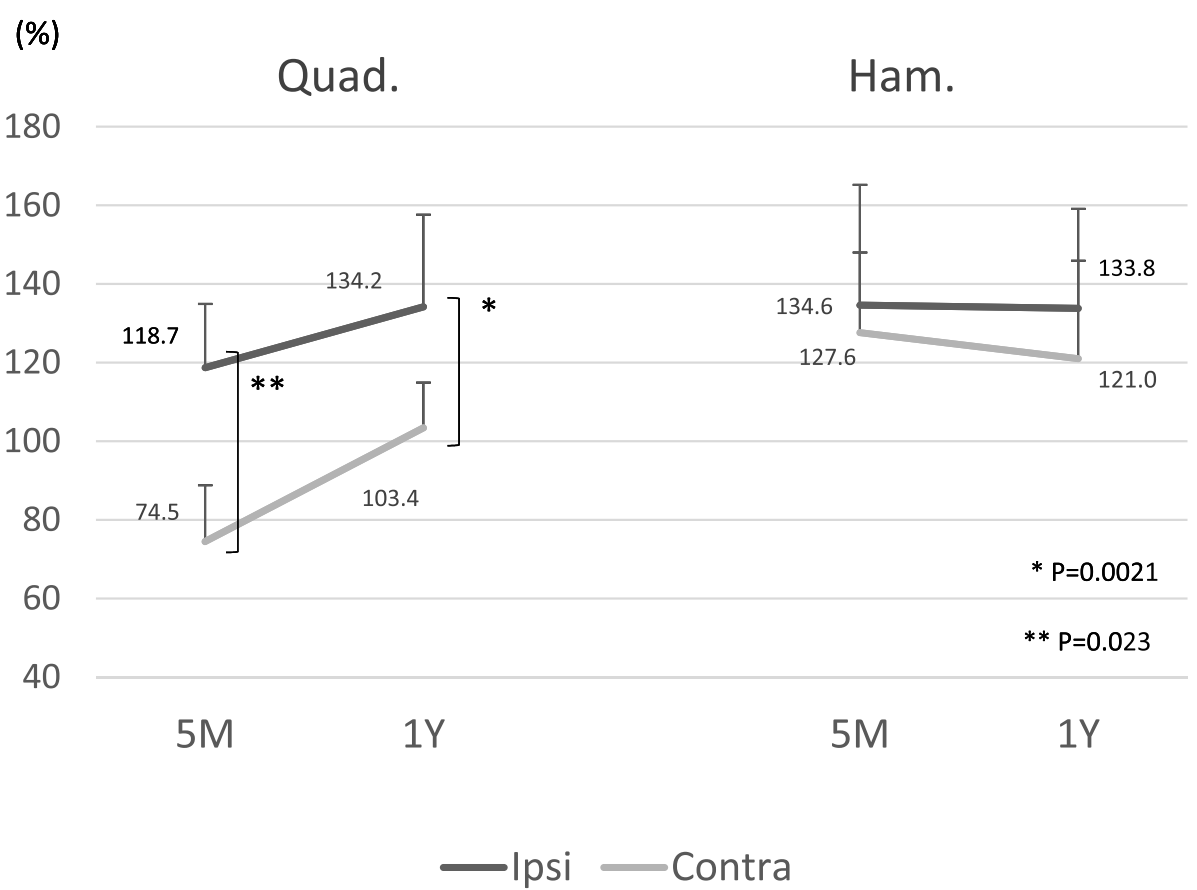

Fig. 3 Muscle strength recovery of the non-reconstructed knees in relation to the preoperative normal knees was investigated. Quadriceps muscle strength was significantly weaker in the C-BTB group at 5 months after surgery (Quad.: $74.5 \pm 14.4 \%$ in the C-BTB vs. $118.7 \pm 27.4 \%$ in the I-BTB; $p=0.0021$, Ham.: $127.6 \pm 20.4 \%$ in the C-BTB vs. $134.6 \pm 26.3 \%$ in the I-BTB; $p=0.60$ ). Moreover, the quadriceps muscle weakness continued at 12 months (Quad.: $103.4 \pm 11.5 \%$ in the C-BTB vs. $134.2 \pm 22.9 \%$ in the I-BTB; $p=0.023$, Ham.: $121.0 \pm 24.9 \%$ in the C-BTB vs. $133.8 \pm 25.7 \%$ in the $\mathrm{I}-\mathrm{BTB} ; p=0.40)$

weakness compared to the preoperative condition. The contralateral ACL reconstruction has limitations in resolving the early return-to-play issue securely.

\section{Acknowledgements}

Co-author of E.U. led the contralateral BTB reconstruction treatment strategy to our institution including surgical technique and rehabilitation protocol. Physiotherapists in our hospital mainly measured pre- and post-operative physical status for the patients.

\section{Authors' contributions}

All authors had substantial contribution to the article. Takaki Sanada (Conceptualization for study design; Data curation; Formal analysis; Investigation; Methodology; Provided and care for the patients; Writing - original draft). Eiji Uchiyama (Original inventor of the treatment concept; Investigation; Provided and care for the patients - review and editing). Hiroshi Iwaso(Investigation; Provided and care for the patients; Scientific advisor - review and editing). Atushi Fukai (Investigation; Provided and care for the patients; - review and editing). The author(s) read and approved the final manuscript.

\section{Funding}

There is no funding source.

\section{Declarations}

\section{Ethics approval and consent to participate}

Institutional review board approval was received for this study.

\section{Consent for publication}

Informed consent of treatment and participation in the investigation were obtains from every patients.

\section{Competing interests}

The all authors declare that they have no conflict of interest. This research did not receive any specific grant from funding agencies in the public, commercial, or not-for-profit sectors.

\section{Author details}

${ }^{1}$ Department of Sports Orthopaedic Surgery, Kanto Rosai Hospital, Kanto Rosai Hospital, 1-1 Kizuki Sumiyoshicho, Nakahara-Ku, Kawasaki, Kanagawa Prefecture 211-8510, Japan. ${ }^{2}$ Inanami Spine and Joint Hospital, Tokyo, Japan. ${ }^{3}$ Faculty of Medical Technology, Teikyo University, Tokyo, Japan.

Received: 15 June 2021 Accepted: 27 August 2021

Published online: 06 October 2021

\section{References}

1. Ardern CL, Webster KE, Taylor NF, Feller JA (2011) Return to sport following anterior cruciate ligament reconstruction surgery: a systematic review and metaanalysis of the state of play. Br J Sports Med 45(7):596-606

2. Beischer S, Gustavsson L, Senorski EH, Karisson J, Thomee C, Samuelsson K et al (2020) Young athletes who return to sport before 9 months after anterior cruciate ligament reconstruction have a rate of new injury 7 times that of those who delay return. J Orthop Sports Phys Ther. 50(2):83-90

3. Benner RW, Shelbourne KD, Freeman H (2011) Infections and patellar tendo ruptures after anterior cruciate ligament reconstruction: a comparison of ipsilateral and contralateral patellar tendon autografts. Am J Sports Med 39(3):519-525

4. Burgi CR, Peters S, Ardern CL, Magill JR, Gomez JS, Reiman MP (2019) Which criteria are used to clear patients to return to sports after primary ACL reconstruction? A scoping review. Br J Sports Med 53:1154-1161 
5. Beynnon BD, Johnson RJ, Naud S, Fleming BC, Abate JA, Brattbakk B (2011) Nichols CE. Accelerated versus nonaccelerated rehabilitation after anterior cruciate ligament reconstruction: a prospective, randomized, double-blind investigation evaluating knee joint laxity using roentgen stereophotogrammetric analysis. Am J Sports Med 39(12):2536-2548

6. Fukuda H, Ogura T, Asai S, Omodani T, et al (2021) Bone-patellar tendonbone autograft maturation is superior to double-bundle hamstring tendon autograft maturation following anatomical anterior cruciate ligament reconstruction. Knee Surg Sports Traumatol Arthrosc 23. https:// doi.org/10.1007/s00167-021-06653-1

7. Ellman MB, Sherman SL, Forsythe B, LaPrade RF, Cole BJ, Bach BR Jr (2015) Return to play following anterior cruciate ligament reconstruction. J Am Acad Orthop Surg 23(5):283-296

8. Kato Y, Chavez J, Yamada S, Hattori S, Takazawa S, Ohuchi H (2019) BetaTricalcium phosphate block for donor site morbidity of the Patella in anterior cruciate ligament reconstruction using bone-patellar tendonbone graft. Knee Surg Related Res 31(2):113-119

9. Lai CCH, Andern CL, Feller JA, Webster KE (2018) Eighty-three per cent of elite athletes return to preinjury sport after anterior cruciate ligament reconstruction: a systematic review with meta-analysis of return to sport rates, graft rupture rates and performance outcomes. Br J Sports Med 52(2):128-138

10. Mastrokalos DS, Springer J, Siebold R, Paessler HH (2005) Donor site morbidity and return to the preinjury activity level after anterior cruciate ligament reconstruction using ipsilateral and contralateral patellar tendon autograft: a retrospective, nonrandomized study. Am J Sports Med 33(1):85-93
11. Schandl K, Horvathy DB, Boros A, Majzik E, Schwarz CM, Csonge L et al (2016) Bone-albumin filling decreases donor site morbidity and enhances bone formation after anterior cruciate ligament reconstruction with bone-patellar tendon-bone autografts. Int Orthop 40(10):2097-2104

12. Schmitt LC, Paterno MV, Hewett TE (2012) The impact of quadriceps Femoris strength asymmetry on functional performance at return to sport following anterior cruciate ligament reconstruction. J Orthop Sports Phys Ther 42(9):750-759

13. Shelbourne KD, Beck MB, Gray T (2015) Anterior cruciate ligament reconstruction with contralateral autogenous patellar tendon graft. Am J Sports Med 4(3):648-653

14. Shelbourne KD, Nitz P (1990) Accelerated rehabilitation after anterior cruciate ligament reconstruction. Am J Sports Med 18(3):292-299

15. Shelboune KD, Trumper RV (1997) Preventing anterior knee pain after anterior cruciate ligament reconstruction. Am J Sports Med 25(1):41-47

16. Shelbourne KD, Urch SE (2000) Primary anterior cruciate ligament reconstruction using the contralateral autogenous patellar tendon. Am J Sports Med 28(5):651-658

17. Shelbourne KD, Vanadurongwan B, Gray T (2007) Primary anterior cruciate ligament reconstruction using contralateral patellar tendon autograft. Clin Sports Med 26(4):549-565

\section{Publisher's Note}

Springer Nature remains neutral with regard to jurisdictional claims in published maps and institutional affiliations.

\section{Submit your manuscript to a SpringerOpen ${ }^{\circ}$ journal and benefit from:}

- Convenient online submission

- Rigorous peer review

- Open access: articles freely available online

- High visibility within the field

Retaining the copyright to your article

Submit your next manuscript at $\boldsymbol{\nabla}$ springeropen.com 